\title{
TEXTURE ANALYSIS BY MEANS OF MODEL FUNCTIONS
}

\author{
TH. ESCHNER* \\ Mining Academy Freiberg (Sa.), Department of Materials Science, POB 47, \\ DO-9200 Freiberg (Sa.), Germany.
}

(12 July 1992)

The conception of texture components is widely used in texture analysis. Mostly it is used to describe the orientation distribution function (ODF) qualitatively, and there are only a few special functions used to provide texture component calculations.

This paper attempts to introduce another model function describing common texture components and giving a compromise between universality and computational efficiency.

KEY WORDS Texture analysis, model function, normal distribution, component fit.

\section{CENTRAL FUNCTIONS}

The very first model ODF introduced into texture analysis has the form (Bunge, 1969):

$$
f^{B G}(g)=S_{0} \exp -\left(\omega / \omega_{0}\right)^{2}
$$

with $\omega$-the orientation distance $\left|g g_{0}^{-1}\right|$. It's series expansion coefficients are expressed as follows:

$$
\begin{aligned}
C_{l}^{m n} & =a_{l} T_{l}^{m n}\left(g_{0}\right) \\
a_{l} & =c_{l}-c_{l+1} \\
c_{l} & =\frac{1}{\sqrt{4 \pi}} S_{0} \omega_{0} \exp \left(-l^{2} \omega_{0}^{2} / 4\right)
\end{aligned}
$$

There are no analytically closed expressions for pole figure values available, so they have to be calculated in terms of a series expansion.

The "standard distributions", introduced in Matthies (1982) supply analytically closed expressions for ODF and pole figure values as well as $C_{l}^{m n}$ coefficients:

$$
\begin{aligned}
f^{S G}(g) & =N(S) \exp (S \cos \omega) \\
S & =\ln (2) /\left[2 \sin ^{2}\left(b_{F W H M} / 4\right)\right] \\
N(S) & =\left[\mathrm{I}_{0}(S)-\mathrm{I}_{1}(S)\right]^{-1} \\
C_{l}^{m, n} & =C_{l}(S) D_{m, n}^{l *}\left(g_{0}^{-1}\right) \\
C_{l}(S) & =N(S)\left[\mathrm{I}_{l}(S)-\mathrm{I}_{l+1}(S)\right] \\
\mathscr{P}_{\vec{h}_{i}}(\vec{y}) & =N(S) \mathrm{I}_{0}(S(1+z) / 2) \exp (S(z-1) / 2) \\
z & =\vec{h}_{i} g_{0} \vec{y}
\end{aligned}
$$

\footnotetext{
* Present address: PTB Braunschweig, 7.4, POB 3345, DW-3300 Braunschweig, Germany.
} 
( $I_{n}$-modified Bessel functions). Note, that due to the presence of $\cos \omega$ this function shows correct behavior near $\omega=\pi$ for big halfwidths, too.

\section{FIBRE TEXTURES}

From (3) a model function describing fibre components has been derived, which is characterized by a crystal direction $\vec{h}$ being parallel to a direction in sample coordinate system $\vec{n}$ :

$$
\begin{aligned}
f^{S F}(g) & =N(S) \exp (S \vec{h} \cdot g \vec{n}) \\
S & =\ln (2) /\left[2 \sin ^{2}\left(b_{F W H M} / 4\right)\right] \\
N(S) & =S / \sinh (S) \\
C_{l}^{m, n} & =\frac{4 \pi}{2 l+1} C_{l} Y_{l m}(\vec{h}) Y_{l n}(\vec{y}) \\
C_{l} & =(2 l+1)(\pi S / 2)^{1 / 2} I_{l+1 / 2}(S) / \sinh S \\
\mathscr{P}_{\vec{h}_{i}}(\vec{y}) & =N(S) \mathrm{I}_{0}\left(S\left(1-z_{1}^{2}\right)^{1 / 2}\left(1-z_{2}^{2}\right)^{1 / 2}\right) \exp \left(S z_{1} z_{2}\right) \\
z_{1} & =\left(\overrightarrow{h_{i}} \cdot \vec{h}\right) \\
z_{2} & =(\vec{y} \cdot \vec{n})
\end{aligned}
$$

\section{GAUSSIAN NORMAL DISTRIBUTION}

Savjolova (1985) gave an expression for a distribution, that was derived from the central limit theorem of probability and gives a very common description of a texture component. All important terms are expressed through the series expansion coefficients $C_{l}^{m n}$, having the following form for the case of a canonical distribution ( 3 parameters $\alpha_{11}, \alpha_{22}, \alpha_{33}$ ):

$$
\begin{aligned}
\left\|C_{l}^{m n}\right\| & =(2 l+1) \exp \left(\left\|b_{m n}^{l}\right\|\right) \\
b_{1+m, 1+n}^{l} & =b_{2 l+1-m, 2 l+1-m}^{l}=-\frac{1}{2}\left((2 m+1) l-m^{2}\right)\left(\alpha_{11}+\alpha_{22}\right)-(l-m)^{2} \alpha_{33} \\
b_{1+m, 3+m} & =b_{3+m, 1+m}=b_{2 l+1-m, 2 l-1-m}=b_{2 l-1-m, 2 l+1-m} \\
& =-\frac{1}{4}((m+1)(m+2)(2 l-m)(2 l-1-m))^{1 / 2}, \quad m=0,1, \ldots, l \\
\text { other } b_{i j} & =0
\end{aligned}
$$

Although this function is of highest interest, it's utilization for texture analysis meets serious complications, mainly due to the high computational effort: all characteristics are expressed in terms of series with coefficients given in form of a matrix exponentiation.

The special case of small halfwidths and a central distribution (5) revealed a very good agreement between (1) and (5), whereas (3) differs about $1 \%$ from (5) (Matthies, Muller, Vinel, 1988). 


\section{A NEW MODEL FUNCTION}

\section{1. $O D F$}

The model function presented below was derived for use in a component analysis program, described by Helming, Eschner (1990), where the calculation of pole figure values is of main interest, so no expressions for $C_{l}^{m n}$ will be given.

For this purpose Eq. (3) was modified: the halfwidth parameter $S$ became directional dependent:

$$
\begin{aligned}
S & =\frac{1}{r^{2}} \\
\frac{1}{r^{2}} & =\frac{\tilde{n}_{x}^{2}}{a^{2}}+\frac{\tilde{n}_{y}^{2}}{b^{2}}+\frac{\tilde{n}_{z}^{2}}{c^{2}} \\
\overrightarrow{\tilde{n}} & =g_{d}^{-1} \vec{n}
\end{aligned}
$$

with $r$ taken from the $r(\vec{n})$-representation of an ellipsoid (main axes $a, b, c)$ and $\vec{n}$ taken from $g g_{0}^{-1}=[\omega, \vec{n}]$ (In some sense $\omega$ and $\vec{n}$ can be considered as functions of $g g_{0}^{-1}$, as will be done further on: $\left.\omega=\omega\left(g g_{0}^{-1}\right), \vec{n}=\vec{n}\left(g g_{0}^{-1}\right)\right) \cdot g_{d}^{-1}$ rotates the ellipsoid out of it's trivial position.

Since

$$
\begin{aligned}
\forall g, g_{d}: g_{d}^{-1} \vec{n}(g) & =\vec{n}\left(g_{d}^{-1} g g_{d}\right) \\
\omega(g) & =\omega\left(g_{d}^{-1} g g_{d}\right)
\end{aligned}
$$

$g g_{0}^{-1}$ can be substituted by $g_{d}^{-1} g g_{0}^{-1} g_{d}$ and the ellipsoid considered to be non-rotated.

With

$$
A_{i i}=(1-\cos \omega) n_{i}^{2}+\cos \omega, i=1, \ldots, 3(x \cdots y \text { resp. })
$$

the diagonal matrix elements of $g_{d}^{-1} g g_{0}^{-1} g_{d}$ in it's $[\omega, \vec{n}]$-representation and $A=1 / a, B=1 / b, C=1 / c$ we get:

$$
\begin{aligned}
-S(1-\cos \omega) & =\alpha_{0}+\alpha_{1} A_{11}+\alpha_{2} A_{22}+\alpha_{3} A_{33} \\
\alpha_{0} & =-\frac{1}{2}\left(A^{2}+B^{2}+C^{2}\right) \\
\alpha_{1} & =\frac{1}{2}\left(-A^{2}+B^{2}+C^{2}\right) \\
\alpha_{2} & =\frac{1}{2}\left(A^{2}-B^{2}+C^{2}\right) \\
\alpha_{3} & =\frac{1}{2}\left(A^{2}+B^{2}-C^{2}\right)
\end{aligned}
$$

and finally ( $\alpha_{0}$ can be omitted due to the normalization)

$$
\begin{aligned}
f\left(g, g_{d}, g_{0}, A, B, C\right) & =N(A, B, C) \exp (-S(\vec{n})(1-\cos \omega)) \\
& =N(A, B, C) \exp \left(\alpha_{1} A_{11}+\alpha_{2} A_{22}+\alpha_{3} A_{33}\right) \\
\vec{n} & =\vec{n}\left(g_{d}^{-1} g g_{0}^{-1} g_{d}\right) \\
\omega & =\omega\left(g_{d}^{-1} g g_{0}^{-1} g_{d}\right) \\
\left|A_{i j}\right| & =g_{d}^{-1} g g_{0}^{-1} g_{d}
\end{aligned}
$$




\subsection{Pole Figures}

It is sufficient to give an expression for ideal pole figures (Matthies, Vinel, Helming, 1987):

From (10) follows:

$$
\mathscr{P}_{\vec{h}}(\vec{y})=\frac{1}{2 \pi} \int_{0}^{2 \pi} f\left(\{\vec{h}, \varphi\}^{-1}\{\vec{y}, 0\}\right) d \varphi
$$

$$
\begin{aligned}
\mathscr{P}_{\vec{h}}(\vec{y}) & =\frac{N(A, B, C)}{2 \pi} \int_{0}^{2 \pi} \exp \left(\alpha_{1} A_{11}+\alpha_{2} A_{22}+\alpha_{3} A_{33}\right) d \varphi \\
\left|A_{i j}\right| & =g^{*}\{\varphi\}^{-1} g^{+} \\
g^{*} & =g_{d}^{-1}\{\vec{h}\}^{-1}=\left|A_{i j}^{*}\right| \\
g^{+} & =\{\vec{y}\} g_{0}^{-1} g_{d}=\left|A_{i j}^{+}\right|
\end{aligned}
$$

With

$$
\begin{aligned}
x= & \alpha_{1} A_{31}^{+} A_{13}^{*}+\alpha_{2} A_{32}^{+} A_{23}^{*}+\alpha_{3} A_{33}^{+} A_{33}^{*} \\
y= & \alpha_{1}\left(A_{11}^{+} A_{11}^{*}+A_{21}^{+} A_{12}^{*}\right)+\alpha_{2}\left(A_{12}^{+} A_{21}^{*}+A_{22}^{+} A_{22}^{*}\right) \\
& +\alpha_{3}\left(A_{13}^{+} A_{31}^{*}+A_{23}^{+} A_{32}^{*}\right) \\
z= & \alpha_{1}\left(A_{11}^{+} A_{12}^{*}-A_{21}^{+} A_{11}^{*}\right)+\alpha_{2}\left(A_{12}^{+} A_{22}^{*}-A_{22}^{+} A_{21}^{*}\right) \\
& +\alpha_{3}\left(A_{13}^{+} A_{32}^{*}-A_{23}^{+} A_{31}^{*}\right)
\end{aligned}
$$

Eq. (12) becomes (see (19)):

With the help of

$$
\begin{aligned}
\mathscr{P}_{\vec{h}}(\vec{y}) & =\frac{N(A, B, C)}{2 \pi} \int_{0}^{2 \pi} \exp (x+y \cos \varphi+z \sin \varphi) d \varphi \\
& =\frac{N(A, B, C)}{2 \pi} \exp (x) \int_{0}^{2 \pi} \exp \left(\sqrt{y^{2}+z^{2}} \cos (\varphi-\tilde{\varphi})\right) d \varphi \\
& =N(A, B, C) \exp (x) \mathrm{I}_{0}\left(\sqrt{y^{2}+z^{2}}\right)
\end{aligned}
$$

$$
\begin{aligned}
g_{d} \vec{h} & =\left(h_{x}^{*}, h_{y}^{*}, h_{x}^{*}\right) \\
g_{d}^{-1} g_{0} \vec{y} & =\left(y_{x}^{+}, y_{y}^{+}, y_{z}^{+}\right)
\end{aligned}
$$

Eqs. (13) and (14) can be rewritten as follows:

$$
\begin{aligned}
x= & \alpha_{1} y_{x}^{+} h_{x}^{*}+\alpha_{2} y_{y}^{+} h_{y}^{*}+\alpha_{3} y_{z}^{+} h_{z}^{*} \\
y^{2}+z^{2}= & x^{2}+\alpha_{1}^{2}\left(1-y_{x}^{+2}-h_{x}^{* 2}\right) \\
& +\alpha_{2}^{2}\left(1-y_{y}^{+2}-h_{y}^{* 2}\right)+\alpha_{3}^{2}\left(1-y_{z}^{+2}-h_{z}^{* 2}\right) \\
& +2 \alpha_{1} \alpha_{2} y_{z}^{+} h_{z}^{*}+2 \alpha_{1} \alpha_{3} y_{y}^{+} h_{y}^{*}+2 \alpha_{2} \alpha_{3} y_{x}^{+} h_{x}^{*} \\
\mathscr{P}_{\vec{h}}(\vec{y})= & N(A, B, C) \exp (x) \mathrm{I}_{0}\left(\sqrt{y^{2}+z^{2}}\right)
\end{aligned}
$$

\subsection{Normalization}

For both transformations $g^{(1)}=g_{1} g$ and $g^{(2)}=g g_{2}$ the jacobians can be shown to equal 1:

$$
\frac{\partial\left(a^{(1)}, \cos \beta^{(1)}, \gamma^{(1)}\right)}{\partial(\alpha, \cos \beta, \gamma)}=\frac{\partial\left(a^{(2)}, \cos \beta^{(2)}, \gamma^{(2)}\right.}{\partial(a, \cos \beta, \gamma)}=1
$$


Hence:

$$
\forall g^{(1)}, g^{(2)}: \int f(g) d g=\int f\left(g^{(1)} g g^{(2)}\right) d g
$$

when integration is performed over the whole Euler space. That means, for purposes of normalization $g_{d}$ and $g_{0}$ can be considered to be $\{0,0,0\}$.

With the help of

$$
\frac{1}{\pi} \int_{0}^{\pi} \exp (x \cos t) \cos (n t)=I_{n}(x)
$$

we obtain

$$
\begin{aligned}
N(A, B, C)^{-1} & =\frac{1}{8 \pi^{2}} \int f(g) d g \\
& =\frac{1}{2} \int_{-1}^{1} d t \exp \left(\alpha_{3} t\right) \mathrm{I}_{0}\left(\frac{A^{2}-B^{2}}{2}(1-t)\right) \mathrm{I}_{0}\left(\frac{C^{2}}{2}(1+t)\right)
\end{aligned}
$$

Probably, this expression cannot be simplified further. Nevertheless this rather simple term can easily be handled in numerical calculations.

\section{EXAMPLES}

Figure 1 shows a few special cases of the model distribution (16) including the case of central function and fibre (cubic/triclinic symmetry). For parameters see Table 1.

In pole figure 1 , distribution (16) is shown for the case of $A=B=C$, when the ellipsoid of halfwidth parameters becomes a sphere and (16) identical to (3). A relation between the full width at half maximum $b_{F W H M}$ and $A(B, C$ resepctively) can be given:

$$
\sqrt{A}=\ln (2) /\left[2 \sin ^{2}\left(b_{F W H M} / 4\right)\right]
$$

Pole figures 2-4 illustrate the case of incomplete fibres with fibre axes in $z-(C<A=B), x-(A<B=C)$ and $y$-direction $(B<A=C)$, respectively.

Pole figure 5 shows a complete fibre with a fibre axis in $x$-direction $(A=0)$. This distribution is equivalent to (4). Equation (21) is valid, too.

In pole figure 6 , a more common case of a distribution (16) is shown $(A \neq B \neq C, A \neq C)$.

In Figure 2, $\mathrm{X}$-ray pole figures of a $\mathrm{Y}$-stabilized $\mathrm{ZrO}_{2}$-coating (obtained at DLR Köln by EB PVD) and recalculated pole figures from a single component texture model are compared. Model parameters can be found in Table 2.

The model reveals quantitatively a fibre direction $\left(\vartheta=30.4^{\circ}, \varphi=251.4^{\circ}\right.$ in sample coordinate system) that was found to coincide with the direction of vapor deposition. 

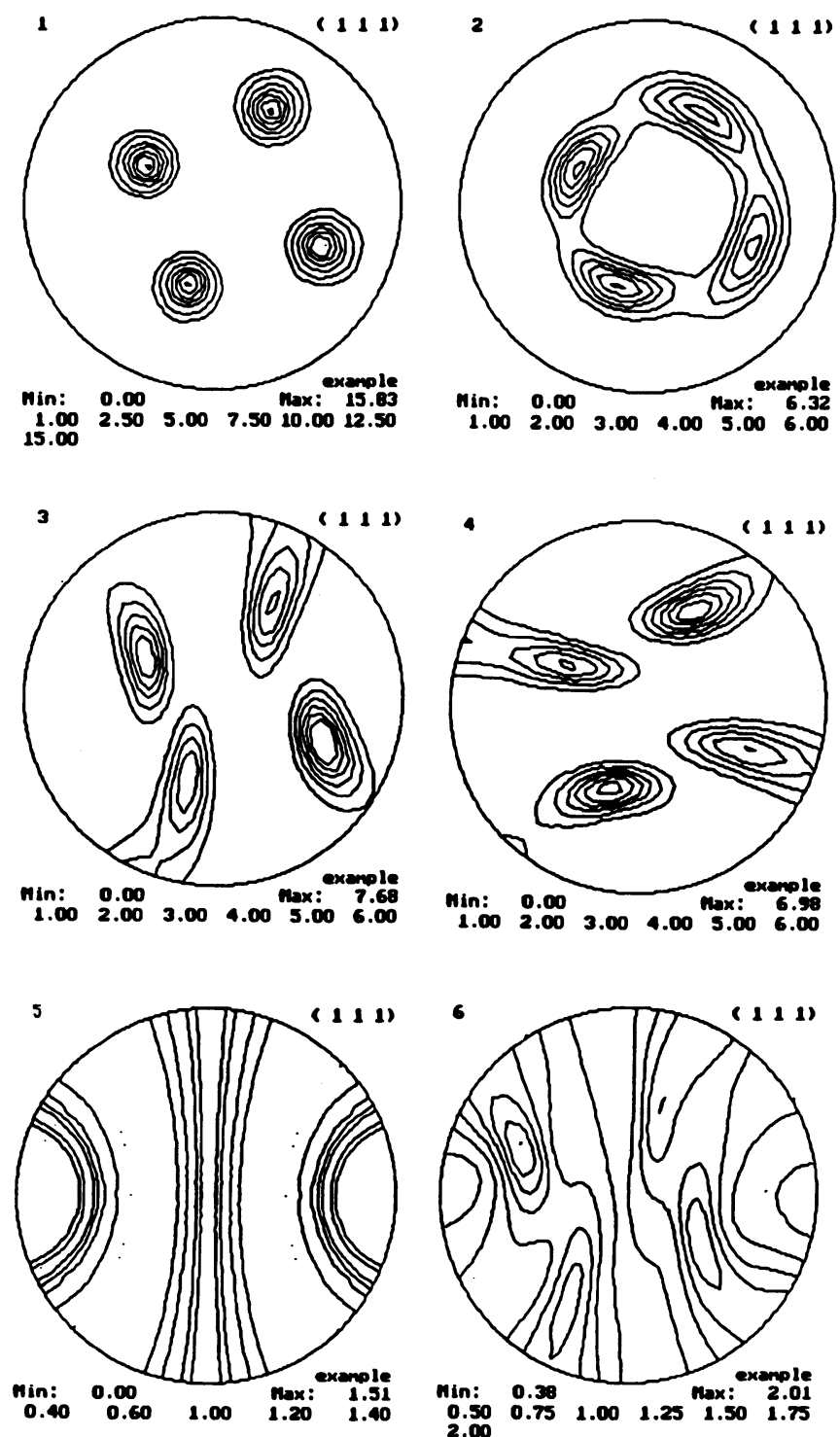

Figure 1 Example pole figures for special values of model parameters according to Table 1. Assumed crystal symmetry: cubic, sample symmetry: triclinic.

Table 1 Parameters of distributions shown in Figure $1, g_{d}=\{0,0,0\}, g_{0}=\left\{10^{\circ}, 10^{\circ}, 10^{\circ}\right\}$

\begin{tabular}{lllllll}
\hline Pole figure & 1 & 2 & 3 & 4 & 5 & 6 \\
\hline$(\mathrm{A}, \mathrm{B}, \mathrm{C})$ & $(8,8,8)$ & $(8,8,2.5)$ & $(2.5,8,8)$ & $(8,2.5,8)$ & $(0,8,8)$ & $(0,2.5,8)$ \\
\hline
\end{tabular}



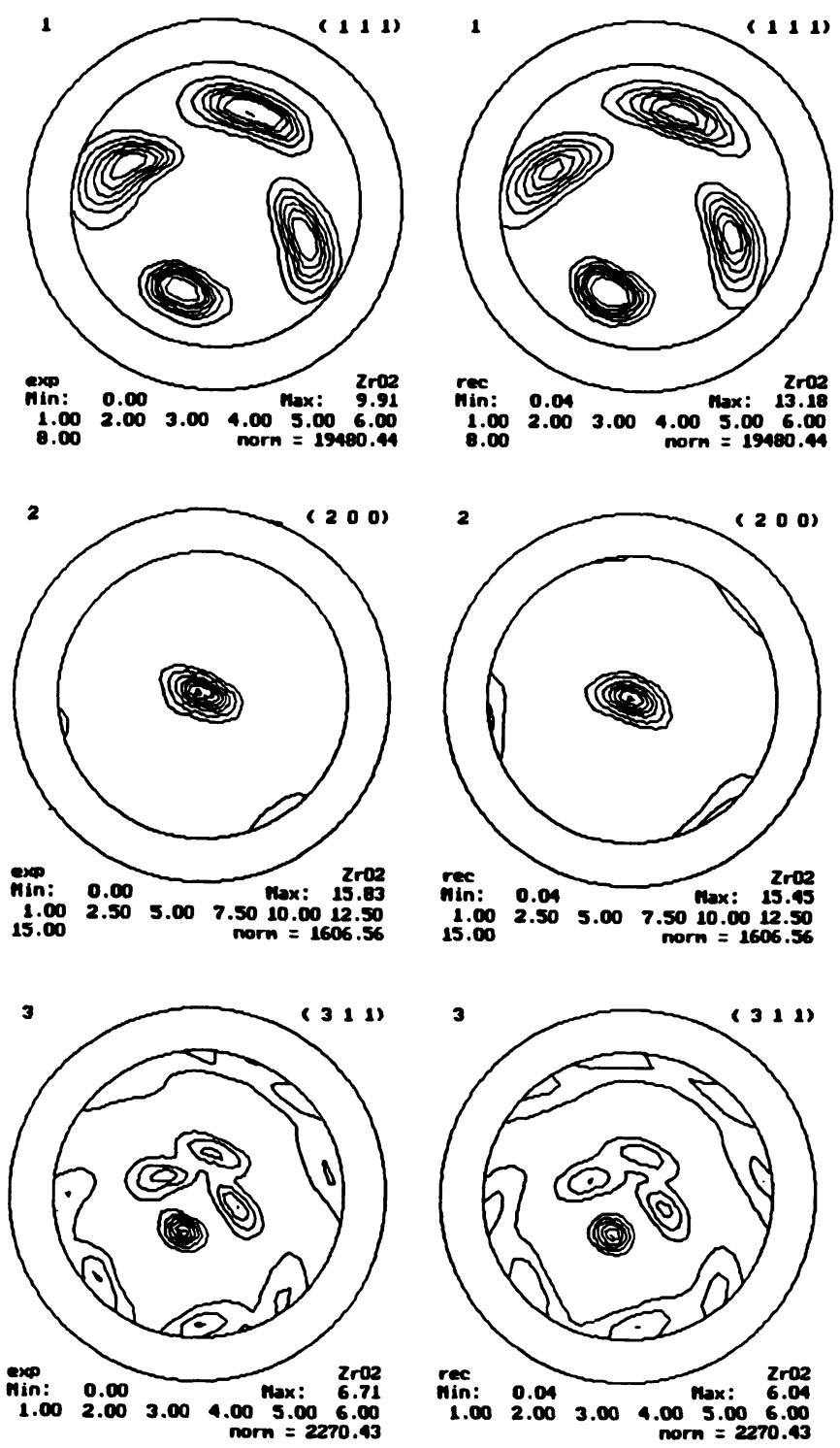

Figure 2 Experimental and recalculated pole figures of a $\mathrm{ZrO}_{2}$-coating. Component parameters can be found in Table 2.

Table 2 Parameters of the single component texture model of a $\mathrm{ZrO}_{2}$-coating

\begin{tabular}{lll}
\hline & Intensity & Parameters \\
\hline background & 0.041 & $(\mathrm{~A}, \mathrm{~B}, \mathrm{C})=(8.096,9.867,3.146)$ \\
texture component & 0.959 & $\left\{\alpha_{0}, \beta_{0}, \gamma_{0}\right\}=\left\{113.5^{\circ}, 1.74^{\circ}, 0.86^{\circ}\right\}$ \\
& & $\left\{\alpha_{d}, \beta_{d}, \gamma_{d}\right\}=\left\{251.4^{\circ}, 30.4^{\circ}, 349.6^{\circ}\right\}$ \\
\hline
\end{tabular}




\section{Acknowledgement}

I would like to thank Mrs. Schwarz, Bergakademie Freiberg, and Mr. Schulz, DLR Köln, for making the experimental data available.

\section{References}

Bunge, H. J. (1969). Mathematische Methoden der Texturanalyse. Akademie-Verlag, Berlin. Helming, K. and Eschner, Th. (1990). Cryst. Res. Technol. 25, K203.

Matthies, S. (1982). Aktuelle Probleme der Quantitativen Texturanalyse, ZfK-480, August 1982. Matthies, S., Muller, J. and Vinel, G. W. (1988). Textures and Microstructures 10, pp. 77-96.

Matthies, S., Vinel, G. W. and Helming, K. (1987). Standard Distributions in Texture Analysis, Akademie-Verlag, Berlin.

Savjolova, T. I. (1985). In: Novye Metody Isledovaniâ Textury Polikristalličeskih Materialov. Metallurgia, Moskva. 